\title{
Review: strong evidence recommends genetic and metabolic testing in subgroups of children with autism
}

Filipek PA, Accardo PJ, Ashwal S, et al. Practice parameter: screening and diagnosis of autism: report of the Quality

Standards Subcommittee of the American Academy of Neurology and the Child Neurology Society. Neurology 2000 Aug

22;55:468-79.

\author{
QUESTION: What evidence supports practice parameters for the surveillance, \\ screening, and diagnosis of children with autism?
}

\section{Data sources}

A panel of experts in autism was convened. Studies in all languages were identified by searching Medline and PsycINFO using the terms autistic, autism, and pervasive; books recommended by members of the panel were also reviewed.

\section{Study selection}

Studies were selected if they were published since 1990, were review papers or meta-analyses developed for DSM$I V$, or were included in the overview of the National Institutes of Health State of the Science Conference on Autism in 1995.

\section{Data extraction}

Studies were assessed for the strength of their evidence: Class I: prospective studies with a description of the study population, inclusion and exclusion criteria specified, adequate sample size; interpretations of evaluations done blinded to outcome; and a satisfactory description of the technology used for the evaluations. Class II: retrospective studies which otherwise met the criteria for class I but did not require blinding. Class III: small cohort or case report studies; or relevant expert opinion, consensus, or survey papers. Recommendations were stratified based on the strength of evidence and called "standards" (high degree of clinical certainty based on Class I studies or overwhelming Class II evidence), "guidelines" (moderate clinical certainty based on $\geqslant 1$ Class II study or a strong consensus of Class III evidence), or "practice options" (uncertain clinical utility based on inconclusive evidence or opinion).

\section{Main results}

2750 studies met the inclusion criteria. 7 practice recommendations were made for routine developmental surveillance and screening of autism with the strength of evidence of a guideline: developmental surveillance should be done at all well child visits from infancy to school age; recommended screening tools include the Ages and Stages Questionnaire, the BRIGANCE Screens, the Child Development Inventories, and the Parents' Evaluations of Developmental Status; because of lack of sensitivity and specificity, the Denver-II and Revised Denver Pre-Screening Developmental Questionnaire are not recommended; more intensive evaluation is required if children fail to meet developmental milestones; siblings of children with autism should be carefully monitored; screening specifically for autism should be done on all children who fail routine developmental surveillance procedures; audiological assessments and lead screening are recommended for children with developmental delay or autism.
6 practice recommendations were made for diagnosis and evaluation; 2 had the strength of evidence of a standard: genetic testing in children with autism should be done in the presence of dysmorphic features or mental retardation, or a family history of Fragile X syndrome or undiagnosed mental retardation; metabolic testing should be done in the presence of such findings as lethargy, cyclic vomiting, early seizures, dysmorphic features, evidence of mental retardation, or questionable adequacy of newborn screening.

\section{Conclusions}

7 practice recommendations for routine developmental surveillance and screening of autism are based on fair evidence. 2 practice recommendations for genetic and metabolic testing in subgroups of children with autism are based on strong evidence.
Sources of funding: National Institutes of Health;AAN

Foundation;Janssen Pharmaceutica; SK Corporation; Abbott Laboratories; Novartis; Athena Neurosciences, Inc.

For correspondence: QSS, American Academy of Neurology, 1080 Montreal Avenue, St Paul, $M N$ 55116, USA. Fax +1 6516952791.

\section{COMMENTARY}

The review by Filipek $e t$ al summarises the consensus of a large and diverse group of autism experts and is based on a systematic review of the current literature. Most of its recommendations are given as "guidelines," as opposed to "standards," reflecting the quality of existing data. Overall, this evidence is below the standards usually expected when proposing a community wide, health related intervention. However, the prevalence of autism is 1-2 out of 1000 , which makes large scale prospective studies of interventions to improve diagnosis extremely difficult.

The authors propose a model of care that advocates greater attention to the early development of children and a greater respect for the concerns of parents. This general framework is supported by available data, and certainly has the potential to increase opportunities for young children with autism to benefit from early intervention. It is worth noting, however, that currently no single instrument exists that is adequate to screen for autism in the general community. The best studied measure, the Checklist for Autism in Toddlers (CHAT), has a sensitivity of only $38 \%$ for autism. ${ }^{1}$ Furthermore, the screening questionnaires proposed for developmental surveillance may be sensitive and specific for general delays, but have never been evaluated for the detection of autism. Primary care providers cannot simply rely on standardised measures to ensure early detection of autism. Filipek et al summarise a number of key "red flags" found during history and direct observation that may identify children in need of more formal assessment. ${ }^{2}$

The authors make the important point that once a child is referred, autism can be reliably diagnosed by an experienced clinician by the time the child reaches 2 years of age. Therefore, considering how best to equip primary care providers with the knowledge and tools needed to detect early signs of autism is certainly worthy of discussion and further research.

Lonnie Zwaigenbaum, MD, $\operatorname{FRCP}(\mathrm{C})$ Children's Hospital at Hamilton Health Sciences Corporation Hamilton, Ontario, Canada

1 Baird G, Charman T, Baron-Cohen S, et al. A screening instrument for autism at 18 months of age: a 6-year follow-up study. J Am Acad Child Adolesc Psychiatry 2000;39:694-702.

2 Filipek PA, Accardo PJ, Baranek GT, et al. The screening and diagnosis of autistic spectrum disorders. J Autism Dev Disord 1999;29;439-84. 